\title{
Experiencia con estocásticos en el segundo grado de educación especial en México
}

\section{Experience with stochastics in the second grade special education in Mexico}

\author{
J. Marcos López Mojica ${ }^{\circledR}$, Jesús A. Larios Trejo², Magdalena Rivera Abrajan ${ }^{3}$
}

Para citar este artículo: López, J. M., Larios, J. A., Rivera, M. (2019). Experiencia con estocásticos en el segundo grado de educación especial en México. Infancias Imágenes, 18(2). [268-277]

\section{Resumen}

En el contexto mexicano la educación especial es una modalidad educativa encargada de atender a niños
Recibido: 12-06-2018 - Aceptado: 09-05-2019

\section{Abstract}

In Mexico, special education is an educational modality dealing with care rendered to children having special education needs due to a disability. This report exhibits the results of the introduction of probability and statistic (stochastic) concepts in a second grade classroom to children with disabilities who were aged between 8 and 10 years. The focus was on recording the teaching process and the analysis of the students' performances before a systematic random situation. Within the interrelation of three theoretical elements (epistemological, cognitive and social), a teaching activity with evaluation forms and observation notes was implemented for the understanding of sample space, probability measure and random variable. The results pertain to the development of stochastic ideas and the use of other mathematical concepts. It is concluded that the treatment of random phenomena in a systematic manner is necessary and mandatory in this educational level to offer comprehensive basic mathematics skills to students. Keywords: mathematics education, special education, teaching strategy. cación especial, estrategia de enseñanza.

1 Doctor en Ciencias con especialidad en Matemática Educativa. Profesor investigador de la Facultad de Matemáticas, Universidad Autónoma de Guerrero. México. Correo electrónico: mojicajm@gmail.com

2 Maestro en Educación. Profesor investigador de la Facultad de Ciencias de la Educación, Universidad de Colima. México. Correo electrónico: jesus_larios@ucol.mx

3 Maestra en Ciencias, área Matemática Educativa. Profesora investigadora de la Facultad de Matemáticas. Universidad Autónoma de Guerrero. México. Correo electrónico: mrivera@uagro.mx 


\section{Introducción}

A casi 25 años de la Declaración de Salamanca (Unesco, 1994), en la que se estableció un marco de acción para la atención de las necesidades educativas especiales (NEE) y la promoción de una Educación Para Todos, surge de manera natural la interrogante sobre el avance en la implementación de esas políticas y marcos legales; de manera particular lo relativo al rubro de la educación. Es decir, ¿cuáles han sido los avances en los sistemas educativos de los 92 países participantes?

En México se comenzó la integración de niños con NEE a las escuelas regulares, además se realizó una reforma al plan de estudios de la licenciatura en educación especial, la cual ahora forma a profesores bajo el modelo de integración educativa (García-Cedillo, 2018). Sin embargo, Flores y García (2016) señalan la escasa comunicación entre los profesores de educación especial y los de educación regular en las aulas para atender en conjunto a los niños que requieren apoyo especializado. Lo anterior es un problema, dado que estos últimos deben ofrecer los contenidos que marca el plan de estudios de la educación básica, tal es el caso de los contenidos en matemáticas.

Al respecto, Bruno, Noda, Aguilar, González, Moreno y Muñoz (2006) señalan que el año 2000 fue declarado Año Mundial de las Matemáticas, en el cual se reflexionó sobre el "cómo poner la matemática al alcance de los niños con necesidades educativas especiales, en particular los que presentan alguna discapacidad" (p. 212); y casi 19 años sigue vigente esa cuestión. Además, existe la carencia de investigaciones que se interesen por los procesos cognitivos o estrategias de enseñanza que permitan el desarrollo de un pensamiento matemático de esta población.

Fernández (2008) y Hegarty (2008) argumentan la necesidad de establecer una relación de la educación especial con otras disciplinas del conocimiento. Más aún, la educación especial debe replantear su modelo de investigación "coherente con las nuevas formas de pensar la realidad" (Fernández, 2008, p. 9). En particular, profundizar sobre el aprendizaje de las matemáticas de niños con NEE, pues es un área poco explorada en la educación especial (Hegarty, 2008).
Los contenidos matemáticos que poco se ha estudiado desde la educación matemática son los estocásticos. La problemática de la enseñanza de los temas de probabilidad y de estadística en distintos niveles educativos en México ha sido documentada (Ojeda, 2006) y una de las consecuencias es la dificultad para identificar aspectos importantes del pensamiento probabilístico, a tomar en cuenta en el planteamiento de actividades que permitan la introducción de la probabilidad en el sistema educativo mexicano.

El descuido de la formación del pensamiento probabilístico de los estudiantes por parte de los docentes, en cualquier nivel educativo, ha causado una limitante a formarlos integralmente; es decir, a prepararlos para enfrentar situaciones deterministas como indeterministas. En consecuencia, se ha generado una sociedad determinista y una deficiencia en el pensamiento crítico.

La necesidad del estudio de la probabilidad y de la estadística es pertinente; desarrollar un pensamiento probabilístico impone un modo distinto de pensar (Fischbein, 1975), porque el pensamiento se abre a lo posible (Piaget, 1982) y en consecuencia se desarrolla un pensamiento crítico. Además, los temas de estocásticos convocan la promoción de otros conceptos matemáticos (Steinbring, 2005) y permiten dotarle de otro sentido por su uso, por lo que establecer una red conceptual para la constitución de un pensamiento matemático que considera la intervención del azar (Heitele, 1975) es indispensable.

La incorporación de estocásticos en la educación requiere de investigaciones acerca de los procesos de su enseñanza y de la comprensión resultante de los educandos en la escuela regular u otros extractos de la educación. Lo anterior para tener un marco de referencia que permita caracterizar el tratamiento de estos en cualquier nivel educativo y no solo eso, sino extenderlo al campo profesional donde se requiera el uso de probabilidad y de estadística.

De lo anterior resulta imperante analizar las conductas manifiestas de los niños del segundo grado de educación especial durante una actividad sobre un fenómeno aleatorio. Estas conductas serán, en todo caso, efecto de las discapacidades particulares 
de que se trate y que permitirán diseñar actividades en función de sus necesidades. Además, se estarían ofreciendo estrategias de enseñanza a profesores que tienen en sus aulas a esta población. La pregunta de interés para el presente documento es: ¿Cuáles son los procesos de enseñanza de estocásticos en el segundo grado de educación especial? En ese sentido, Gaviria, Torres y Torres (2014) exponen una necesidad de herramientas didácticas que estén adaptadas a la población que presenta alguna discapacidad. Pues algunos profesores no están capacitados para el diseño y gestión de su clase con un enfoque inclusivo.

La educación especial en México es una modalidad educativa creada para atender a poblaciones que presentan necesidades educativas especiales asociadas o no a una discapacidad (Romero y García, 2013). La Ley General para las Personas con Discapacidad (LGPD, 2008) la define como un conjunto de servicios especializados puestos a disposición de las personas con discapacidad para lograr los fines de la educación. Esta modalidad educativa está separada de los espacios de la educación regular, pero ambas forman parte de la educación básica del país.

Los servicios que ofrece la educación especial se agrupan en: de orientación, de apoyo y escolarizados. Dentro de los últimos se encuentran los Centros de Atención Múltiple (CAM), los cuales se encargan de escolarizar a niños que presentan alguna discapacidad o discapacidad múltiple, o bien aquellos que, por otras circunstancias, no pueden ser integrados a las escuelas regulares (Secretaría de Educación Pública, 2004), en este espacio fue donde se desarrolló la investigación.

\section{Elementos teóricos}

Para analizar el proceso de enseñanza de estocásticos se tomó la propuesta de Tres Ejes Rectores (Ojeda, 1994) para la comprensión de ideas fundamentales de estocásticos (Ojeda, 2006): epistemológico, cognitivo y social. Se partió de que la intuición se deriva de la experiencia (Fischbein, 1975) y de que surgen esquemas que compensan ausencias o limitaciones en el desarrollo de niños con deficiencias preceptúales, cognitivas o motoras (Vygotski, 1997).

\section{Elementos epistemológicos}

Para este eje interesó la propuesta de Heitele (1975) sobre 10 ideas fundamentales de estocásticos como guía para un currículo en espiral. Para el autor, una idea fundamental es "[...] aquella que proporciona al individuo modelos explicativos tan eficientes como sea posible" (p. 188). Argumenta que el tratamiento de las ideas fundamentales debe partir de un plano intuitivo y arribar a un plano formal, de manera que se garantice continuidad en la educación. Para este informe de investigación nos centramos en: espacio muestra, como el conjunto de todos los posibles resultados de un fenómeno aleatorio; medida de probabilidad, con la asignación de un valor a nuestra creencia dado un posible resultado del fenómeno aleatorio; variable aleatoria, con la relación entre el conjunto de posibles resultados y un subconjunto de los números reales.

\section{Elementos cognitivos}

Como se adelantó, se tomó la acepción de intuición propuesta por Fischbein (1975). El autor la define como un conocimiento que se deriva de la experiencia, de recuperación inmediata, sintética y que se extrapola. Señala que en la formación de intuiciones probabilísticas es necesario considerar lo incierto y conectarlo con la acción por medio de frecuencias relativas; así se establecerá un comportamiento de la situación aleatoria caracterizado como "más probable", "menos probable" o "igualmente probable". La probabilidad es apropiada para el estudio de esas intuiciones debido a su enfoque frecuencial, pues está determinada por la acción y que junto con la observación de los fenómenos naturales se puede desarrollar una base intuitiva.

Además, interesaron los esquemas compensatorios (Vygotski, 1997), estos son procesos cognitivos que permiten superar una ausencia o limitación, reestructurando el pensamiento del niño ante la discapacidad.

\section{Elementos de orden social}

Debido a la naturaleza del problema de investigación, se asume la postura de que la discapacidad es un constructo social. Según Vygotski (1997), no es la característica del individuo la que limita su 
desarrollo, sino que es el medio en el que este se desenvuelve. Para Guajardo (2010) "[...] tiene que ver con el desempeño individual en función a las expectativas del entorno y de acuerdo a lo esperado según edad, sexo y grupo social" (p. 109). Por lo tanto, interesan las relaciones que se establezcan entre el individuo con discapacidad y su contexto (Maturana, 2003).

Por otra parte, Steinbring (2005) plantea una relación entre la naturaleza epistemológica del concepto matemático y su significado constituido socialmente, en las interacciones en el aula. El autor argumenta que para la adquisición de un concepto matemático es necesaria la interacción entre el contexto de referencia en que se implica al objeto, el signo y el concepto matemático. Por lo tanto, propone un triángulo relacional para constituir el significado del conocimiento matemático.

\section{Método, instrumento y técnicas}

La experiencia de la enseñanza de estocásticos siguió los lineamientos del órgano operativo y de la célula de análisis de la enseñanza (Ojeda, 2006). El primero organiza los espacios en los cuales se llevó a cabo la investigación, para arribar al aula y así caracterizar el proceso de enseñanza. La célula pone en juego los elementos conceptuales para su aplicación mediante criterios de análisis en cada escenario, de esa manera se pudo caracterizar y contextualizar el proceso de enseñanza.

Los criterios de análisis (Ojeda, 2006) fueron: ideas fundamentales de estocásticos, otros conceptos matemáticos, recursos semióticos, términos para referirse a estocásticos y esquemas compensatorios.

El espacio en el que se aplicó la actividad de enseñanza de estocásticos se denominó aula alterna (Ojeda, 2006), caracterizada así por ser una alternativa a la enseñanza tradicional. En ella confluyen la docencia y la investigación, de manera que la docente se inicia en la indagación de su propia enseñanza (Ojeda, 2006), de las relaciones entre el contenido matemático y las producciones de los niños. Además, en el aula alterna interactúan tanto la docente titular como el investigador, pues ambos realizan las sesiones de enseñanza con la intervención de uno u otro, según sea necesario. Para esta actividad de enseñanza la docente titular aplicó las estrategias y el investigador solo fue observador.

Los métodos fueron la bitácora y la experienciación (Maturana, 2003). Los instrumentos de registro de datos fueron los guiones de estrategia de enseñanza y las hojas de control; las técnicas de registro de datos fueron la escritura con lápiz y papel, o en pizarrón, la videograbación y su transcripción.

\section{Participantes}

La actividad se dirigió a siete niños (8-10 años) con diversos niveles y tipos de discapacidades (tabla 1) de un Centro de Atención Múltiple de la Ciudad de México, institución que ofrece servicios educativos a niños con discapacidad. La actividad se aplicó en una sesión de 40 minutos en los tiempos institucionales establecidos.

\section{La situación de referencia}

La actividad de enseñanza "Calcula y gana" se deriva del libro de texto para el segundo grado de educación primaria regular (Secretaría de Educación Pública, 2006, p. 32). Se le aplicaron los criterios de análisis (Ojeda, 2006) y se identificaron las siguientes ideas fundamentales de estocásticos: independencia, pues el resultado del lanzamiento de un dado en nada afecta el resultado del lanzamiento del otro; espacio muestra, con la variación

Tabla 1. Características individuales en el aula alterna del segundo grado.

\begin{tabular}{|c|c|c|c|c|c|c|c|}
\hline & \multicolumn{4}{|c|}{ Síndrome de Down } & \multicolumn{2}{|c|}{ Discapacidad Intelectual } & \multirow{2}{*}{$\begin{array}{c}\begin{array}{c}\text { Síndrome } \\
\text { Weber }\end{array} \\
M\end{array}$} \\
\hline & B & $\mathbf{E}$ & $\mathbf{J}$ & $\mathbf{C}$ & I & $\mathbf{K}$ & \\
\hline Casos & $\begin{array}{l}\text { Mujer } \\
8 \text { años }\end{array}$ & $\begin{array}{l}\text { Hombre } \\
8 \text { años }\end{array}$ & \begin{tabular}{|l} 
Hombre \\
8 años
\end{tabular} & $\begin{array}{l}\text { Hombre } \\
11 \text { años }\end{array}$ & $\begin{array}{l}\text { Mujer } \\
8 \text { años }\end{array}$ & $\begin{array}{l}\text { Mujer } \\
11 \text { años }\end{array}$ & $\begin{array}{l}\text { Mujer } \\
9 \text { años }\end{array}$ \\
\hline Comunicación & Oralizada & $\begin{array}{l}\text { Sonidos gutu- } \\
\text { rales }\end{array}$ & $\begin{array}{l}\text { Palabras ais- } \\
\text { ladas }\end{array}$ & $\begin{array}{l}\text { Sonidos gutu- } \\
\text { rales }\end{array}$ & $\begin{array}{l}\text { Palabras ais- } \\
\text { ladas }\end{array}$ & $\begin{array}{l}\text { Sonidos gutu- } \\
\text { rales }\end{array}$ & Oralizada \\
\hline
\end{tabular}


de resultados al lanzar los dos dados; y de variable aleatoria, de manera cualitativa con la frecuencia relativa de cada evento. La docente pidió a los niños y niñas la reproducción en una hoja cuadriculada de los datos de una tabla numerada del 1 al 100. La aplicación de los criterios de análisis de muestra en la tabla 2.

La actividad consistió en trabajar en grupos utilizando dados distinguibles (azul y rojo) por el color y el tamaño, ordinarios (cúbicos, con puntos en las caras) (figura 1). Se empleó una tabla para registrar el número de puntos obtenidos en cada lanzamiento, los dos dados se lanzaron consecutivamente; los puntos del dado azul indicaban unidades y los puntos del dado rojo indicaban decenas; se demandó la suma de los valores obtenidos y avanzar según el resultado de la suma, partiendo del valor inicial, en una cuadricula grande realizada por la docente para el registro en grupo. Gana quien arribe primero a la casilla 100.

Los alumnos, para saber si llegaban o se aproximaban a 100, tenían que realizar la suma de las posiciones dadas y la obtenida mediante el conteo propuesto por la docente, utilizando fichas de dos colores, verdes y rojas, para las cantidades. Como se podrá notar, si bien la actividad refiere a la introducción del valor posicional, esta implica un fenómeno aleatorio el cual no puede ser desaprovechado. De esta forma, se le estaría ofreciendo al estudiante una formación integral; es decir, una preparación ante situaciones deterministas como indeterministas. Además, se le está presentando la idea de valor posicional con otro uso.

\section{Resultados de la enseñanza en el aula alterna}

En la actividad de enseñanza "Calcula y gana" dos niños con síndrome de Down (B y J) identificaron algunos casos del espacio muestra, $\mathrm{K}$ (discapacidad intelectual) y $\mathrm{M}$ (síndrome Weber) asignaron valores a los puntos de los dados, lo cual permitió un acercamiento a la variable aleatoria. En las siguientes intervenciones, se emplea la inicial del nombre del alumno y $\mathbf{P}$ para la docente titular.

Tabla 2. Caracterización de la actividad Calcula y Gana

\begin{tabular}{|c|c|c|c|c|}
\hline Situación y contexto & $\begin{array}{c}\text { Ideas } \\
\text { fundamentales de } \\
\text { estocásticos }\end{array}$ & $\begin{array}{l}\text { Otros conceptos } \\
\text { matemáticos }\end{array}$ & $\begin{array}{l}\text { Recursos } \\
\text { semióticos }\end{array}$ & $\begin{array}{l}\text { Términos } \\
\text { empleados }\end{array}$ \\
\hline $\begin{array}{l}\text { Juego con lanzamientos sucesivos } \\
\text { de dos dados distinguibles; asig- } \\
\text { nación de tantas decenas como } \\
\text { puntos se obtengan con uno de los } \\
\text { dados y de tantas unidades como } \\
\text { puntos se obtengan en el otro. }\end{array}$ & $\begin{array}{l}\text { Espacio muestra, in- } \\
\text { dependencia, una va- } \\
\text { riable aleatoria. }\end{array}$ & $\begin{array}{l}\text { Valor posicional, nú- } \\
\text { meros naturales, or- } \\
\text { den, conteo, adición } \\
\text { y sustracción. }\end{array}$ & $\begin{array}{l}\text { Lengua natural, sig- } \\
\text { nos numéricos, gráfi- } \\
\text { ca, tablas, figuras. }\end{array}$ & $\begin{array}{l}\text { Llegar a, acertar, } \\
\text { avanzar, avanzar } \\
\text { hasta, tirar, gana, } \\
\text { salieron, cuántos. }\end{array}$ \\
\hline
\end{tabular}

Fuente: Ojeda (2006).
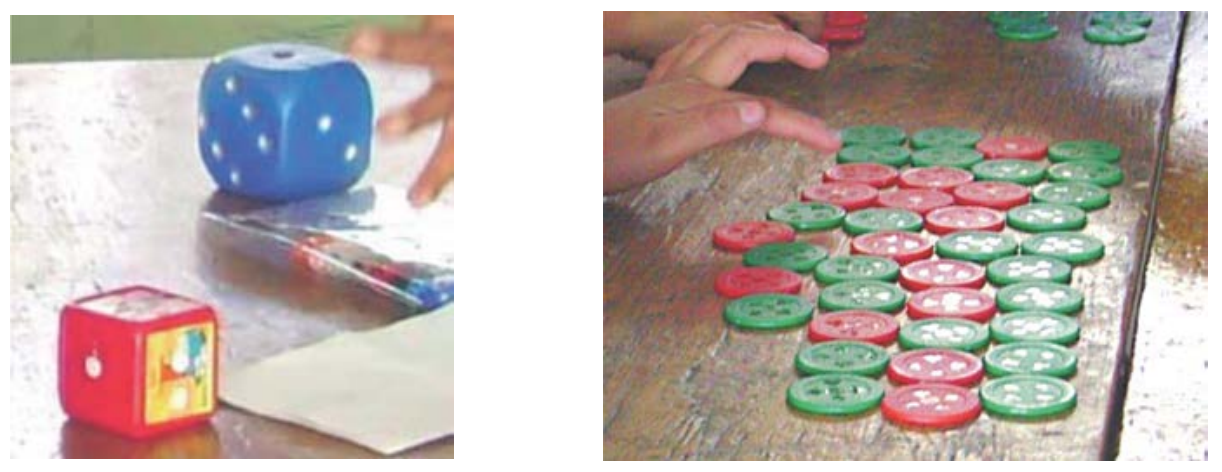

Figura 1. Material empleado en la actividad "Calcula y gana". 


\section{Espacio muestra}

Se tuvo un acercamiento al espacio muestra al identificar algunos posibles resultados, por ejemplo, cuando la docente pedía que se lanzaran los dados, la pregunta "¿cuántas bolitas cayeron?" centró la atención del alumno en la cantidad de "puntitos" de la cara del dado que quedó hacia arriba; además, la pregunta orientó la acción del alumno a la asignación de un numeral a la cantidad de "bolitas", de donde se aproximó al espacio muestra respectivo.

\begin{tabular}{|c|l|}
\hline$[110]$ P: & ¿Cuántos cayeron? ¿Cuántas bolitas hay? \\
\hline$[111]$ I: & ¡Uno! [Gritando]. \\
\hline$[112] \mathrm{E}:$ & Uno [sonriendo]. \\
\hline$[113]$ P: & Uno [levantando el dedo índice]. \\
\hline$[385]$ P: $:$ & $\begin{array}{l}\text { iCayó así! [Mostrando la cara del dado, 5]... } \\
\text { ¿Cuánto cayó? }\end{array}$ \\
\hline$[386]$ M: $:$ & ¡Cinco! [Extendiendo la mano]. \\
\hline$[387]$ P: & Cinco, ¿verdad?... \\
\hline $\begin{array}{r}\text { [388] K, } \\
\text { M e l: }\end{array}$ & ¡Sí! \\
\hline
\end{tabular}

Según los elementos teóricos, las acciones solicitadas por la docente, como el lanzamiento de los dados e identificación de los resultados, sentarían bases intuitivas (Fischbein, 1975) si se realizan de manera repetida.

\section{Independencia}

El acercamiento a esta idea fundamental se da con la repetición (Fischbein, 1975) del lanzamiento de los dados. Se puede notar un señalamiento al fenómeno aleatorio [94, 96]. Sin embargo, no ocurrieron expresiones del tipo "No importa lo que caiga en un dado, cualquiera de las seis caras pueden caer en el otro" que refirieran a la idea de independencia de manera declarada:

\begin{tabular}{|l|l|}
\hline [94] P: & $\begin{array}{l}\text { Estamos en estos [señalando los números selec- } \\
\text { cionados por los niños], con estos tres vamos } \\
\text { [valores iniciales] a trabajar ¿ sí? Vamos a hacer } \\
\text { lo posible por llegar al número cien. ¿Cómo lo } \\
\text { vamos a hacer? Vamos a tirar nuestros dados... }\end{array}$ \\
\hline$[95] \mathrm{M:}$ & iAy! [Molesta]. \\
\hline [96] P: & $\begin{array}{l}\text { Si no llegamos a ese número, no importa, hasta } \\
\text { donde lleguemos. Vamos a tirar nuestro dado y } \\
\text { lo vamos hacer; son cuatro [niños]. Lo vamos } \\
\text { hacer por parejas; entonces, cada uno va a tirar, } \\
\text { primero tira uno y después el otro, primero una } \\
\text { pareja y después la otra; [refiriéndose a M] ¿qué } \\
\text { color quieres? }\end{array}$ \\
\hline
\end{tabular}

\section{Variable aleatoria}

Con acciones como asignar valores numéricos a cada uno de los "puntitos de los dados" [8, 10] (Fischbein, 1975; Heitele, 1975), se sugiere el uso de nociones de esta idea fundamental. Nótese que la docente desconoció que se trata de dados ordinarios e incluyó valores que son imposibles de obtener: 0, 7, 8, 9 en un solo lanzamiento [6]; en consecuencia, el espacio muestra quedó desdibujado. Ningún niño se percató de esto. No obstante, los dígitos se utilizaron cuando se contaban las fichas.

\begin{tabular}{|c|c|}
\hline [6] P: & $\begin{array}{l}\text { Decena significa que vamos a tener grupos de diez } \\
\text { cosas; no importan las cosas que tengamos: perros, } \\
\text { gatos, fichas, lápices. ¡No importa!, pero van a ser } \\
\text { [...]. El dado rojo [tomándolo en las manos] nos va } \\
\text { a indicar el grupo de los dieces, el grupo de la de- } \\
\text { cena; y este [señalando el dado azul] nos va a indi- } \\
\text { car el grupo de las unidades, del uno al nueve, del } \\
\text { cero al nueve, ehhh... Recuerden que cada una de } \\
\text { las bolitas va a valer uno en este dado azul; ;uno! } \\
\text { A ver, todos: juno! [Moviendo el dedo índice]. }\end{array}$ \\
\hline [7] T: & $\begin{array}{l}\text { ¡Uno! [Moviendo el dedo índice de la mano } \\
\text { derecha]. }\end{array}$ \\
\hline & $\begin{array}{l}\text { [...] Ehhh, cada bolita corresponde a uno. En este } \\
\text { dado [tomando el dado rojo], que es el grupo de } \\
\text { los dieces, el grupo de la decena, [cada una de las } \\
\text { bolitas] va a valer diez, jasí! [mostrando todos los } \\
\text { dedos de ambas manos]. }\end{array}$ \\
\hline [9] T: & ¡Diez! [Levantando las manos y agitándolas]. \\
\hline [10] P: & $\begin{array}{l}\text { [...] Diez cosas, pero cada una de las bolitas va a } \\
\text { valer diez, cada una de las bolitas vale diez, ¿de } \\
\text { acuerdo? }\end{array}$ \\
\hline
\end{tabular}

Se utilizaron soportes concretos (fichas) para los valores de la variable aleatoria (figura 2). Un aspecto que hubiera convenido tomar en cuenta es la correspondencia de color entre dados y fichas para promover el uso de esquemas compensatorios del alumno. Sin embargo, el uso del soporte concreto favoreció centrar la atención en la actividad y promovió el uso de la memoria de trabajo (Vygotski, 1997), ante la necesidad de recuperar la cantidad de fichas según los puntos de los dados.

La asignación de la cantidad de fichas en correspondencia al resultado de los "puntitos" de las caras de dos dados distinguibles, además de la organización de las fichas en filas de 10 (ver figura 2), permitió darle otro uso a la idea de valor posicional, a pesar de la ausencia de distinguir de manera declarada al aspecto aleatorio que supone el material concreto utilizado. 

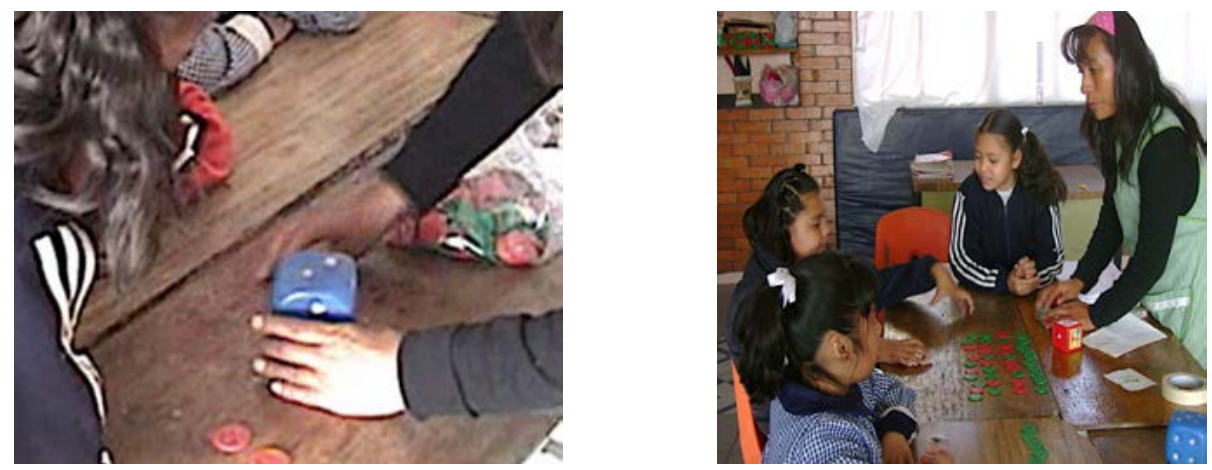

Figura 2. Asignación de fichas según los "puntitos" de cada dado.

\section{Adición de números naturales}

Se pudo notar que, dada la situación de enseñanza, se recurrió a otros conceptos matemáticos, en este caso la adición. De manera explícita, la docente agregó a una colección de fichas en cantidad correspondiente a una cifra dada la obtenida del lanzamiento de los dados y pidió a los niños indicar la cantidad total de fichas (figura 3). La docente indicó sumar, juntar todas las fichas a manera de estimular la adición; nótese el énfasis en expresiones con sus manos a manera de unir los conjuntos de fichas. Lo anterior es dado al tipo de comunicación que se estableció con los estudiantes, pues recordemos la característica particular de cada uno señalada en la tabla 1.

La actividad desarrollada en el aula alterna distinguió entre signo, objeto y concepto, lo cual es necesario para la constitución del concepto (Steinbring, 2005), en particular de estocásticos.

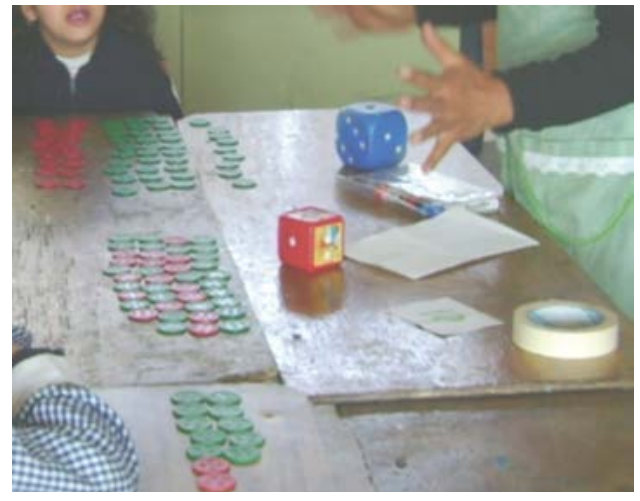

Figura 3. Agregación de dos colecciones de fichas y la cantidad total obtenida.
La situación de referencia provocó una red conceptual (Steinbring, 2005) que implicaba no solo la distinción de las nociones señaladas de estocásticos (figura 4), sino también la de otros conceptos matemáticos como lo fue la adición y el valor posicional.

La red conceptual se establece con la relación entre los triángulos epistemológicos para cada concepto matemático. Por ejemplo, a partir de la relación entre objeto 1 , signo 1 y concepto 1 , se puede desprender el triángulo con relaciones objeto 1 , signo 2 y concepto 2 ; y a la vez se puede tener el triángulo cuyas relaciones serían objeto 1 , signo 3 y concepto 3 (figura 4).

Para el caso particular de la actividad "calcula y gana", las estrategias de la docente permitieron dicha distinción. Por ejemplo, la situación de referencia fue la colección de fichas en unidades y decenas según la variedad de resultados del

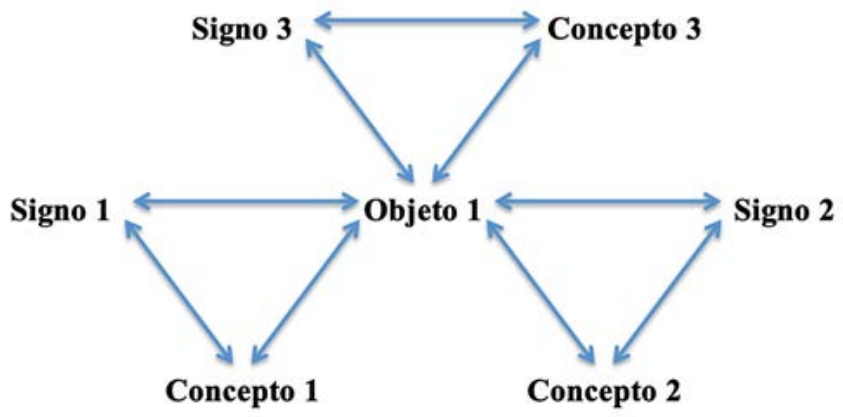

Figura 4. Red conceptual para la naturaleza epistemológica del concepto matemático.

Fuente: Steinbring (2005). 
lanzamiento de los dados; el signo correspondiente fue la escritura en tabla de doble entrada de los numerales correspondientes; esa asignación promovió la idea de variable aleatoria (figura 5).

También se tuvo la distinción para el espacio muestra, ya que las caras de los dados indicaban los posibles resultados del fenómeno aleatorio del lanzamiento de dados. Los numerales se utilizaron para identificar la cantidad de puntos de cada cara del dado (figura 6).

En el caso del concepto de valor posicional, la situación de referencia consistió en la constitución de cantidades de dos cifras utilizando dados y fichas, para formar colecciones de unidades y decenas; como signo se utilizó una tabla cuadriculada numérica con la serie del 1 al 100 (figura 7).
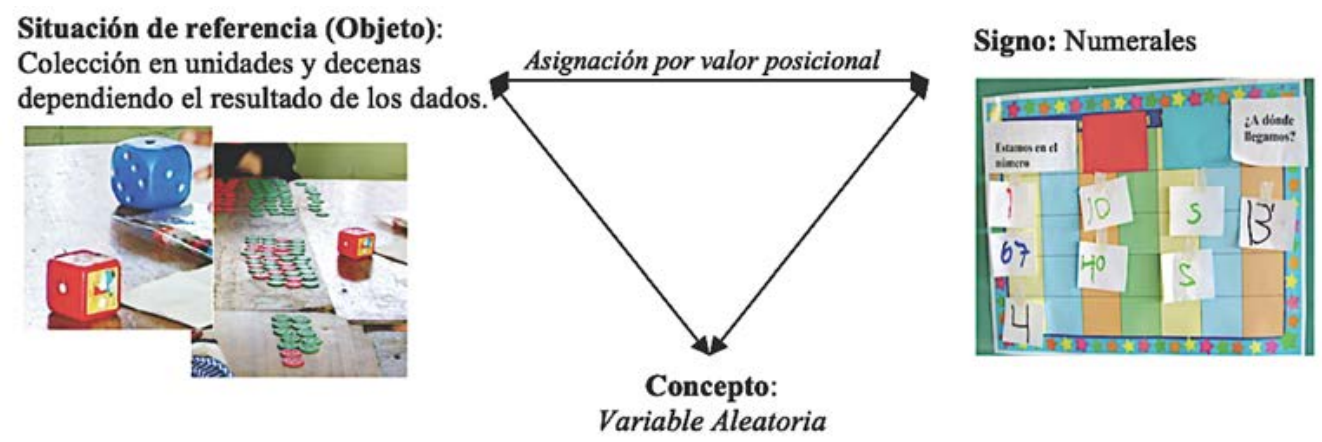

Figura 5. Triángulo epistemológico para el concepto de variable aleatoria.

Fuente: Steinbring (2005).

Situación de referencia (Objeto): Posibles resultados $[1,2,3,4,5,6]$ Cantidad de "puntos".
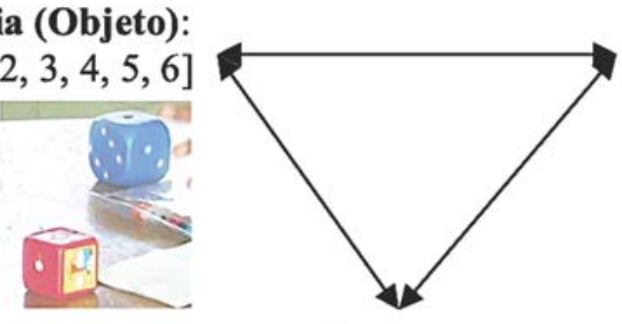

Signo: Numerales

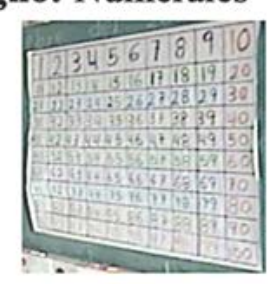

\section{Concepto:}

Espacio muestra

Figura 6. Triángulo epistemológico para el concepto de espacio muestra.

Fuente: Steinbring (2005).

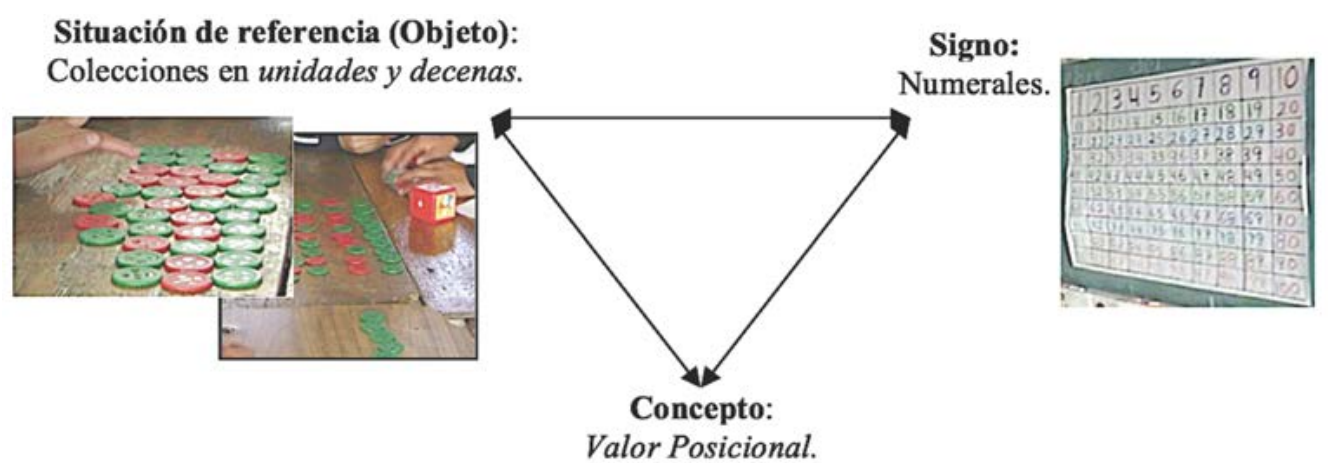

Figura 7. Triángulo epistemológico para el concepto de valor posicional.

Fuente: Steinbring (2005). 


\section{Conclusiones y comentarios}

Sobre los resultados se puede decir que, de los siete niños del segundo grado de primaria especial de un Centro de Atención Múltiple en condiciones de síndrome de Down, síndrome Weber y discapacidad intelectual, se permite recomendar la enseñanza de la probabilidad y de la estadística en ese nivel educativo.

En el desarrollo de la actividad se tuvo un acercamiento a las nociones de espacio muestra, medida de probabilidad y asignación de valores de algunos eventos por parte de los niños con discapacidad intelectual y síndrome de Down. Lo anterior se logró con las acciones como lanzar los dados e identificar los resultados, la correspondencia entre los puntos de las caras superiores de los dados y la cantidad de fichas correspondientes, así como el registro en tabla de las fichas con el numeral correspondiente. Estas acciones están relacionadas con la memoria de trabajo, la cual permitió que estos alumnos tuvieran el acercamiento a las nociones matemáticas señaladas.

La promoción en actividades de enseñanza de experiencias con situaciones que impliquen fenómenos aleatorios, favorecen el desarrollo de intuiciones (Fischbein, 1975). Cuantas más experiencias sobre estocásticos tengan los niños desde edades tempranas, más se fomentará el desarrollo de las ideas fundamentales (Heitele, 1975) y se prevendrán los sesgos en el pensamiento probabilístico (Tversky y Kahneman, 1982).

Lo anterior cumple también para la población de educación especial, ya que de esa manera se le ofrece una educación integral. Además, los estocásticos son promotores de una red conceptual (Steinbring, 2005), es decir, aquellos convocan otros conceptos matemáticos como en este caso valor posicional.

En lo que respecta a la práctica docente, la profesora propuso y desarrolló más actividades en las que se trataba un fenómeno aleatorio. Además, dada su experiencia con la población se pudo señalar el tipo de material, el tipo de preguntas e instrucciones a emplear en el aula.

Se puede concluir que no solo es posible el tratamiento de la probabilidad en la educación especial, sino necesario para proporcionar una formación matemática integral, esta debería de promover el uso de los esquemas compensatorios, pues ellos permiten desarrollar el pensamiento de los niños con discapacidad.

\section{Referencias}

Bruno, A., Noda, M., Aguilar, R., González, C., Moreno, L. y Muñoz, V. (2006). Análisis de un tutorial inteligente sobre conceptos lógico-matemáticos en alumnos con síndrome de Down. Revista Latinoamericana en Matemática Educativa, 9(2), 211-226. Recuperado de http://www. scielo.org.mx/scielo.php?script=sci_arttext\&pi$\mathrm{d}=$ S1665-24362006000200003

Fernández, J. M. (2008). La investigación en educación especial. Líneas temáticas y perspectivas de futuro. Perfiles Educativos, XXX(119), 7-32. Recuperado de https://www.redalyc.org/articulo.oa?id=13211156002

Fischbein, E. (1975). The Intuitive Sources of Probabilistic Thinking in Children. Dordrecht, Países Bajos: D. Reidel.

Flores, V. J. y García, I. (2016). Apoyos que reciben estudiantes de secundaria con discapacidad en escuelas regulares: ¿corresponden a lo que dicen las leyes? Educación, 2(40), 1-20. https:// doi.org/10.15517/revedu.v40i2.15851

García-Cedillo, I. (2018). La educación inclusiva en la Reforma Educativa de México. Revista de Educación Inclusiva, 11(2), 49-62. Recuperado de http://www.revistaeducacioninclusiva.es/index.php/REl/article/view/373

Gaviria, Y. R., Torres, J. J. y Torres, E. (2014). Una propuesta inclusiva para la representación geométrica de los poliedros con población en condición de discapacidad visual. Infancias Imágenes, 13(2), 111-125. https://doi. org/10.14483/udistrital.jour.infimg.2014.2.a09

Guajardo, E. (2010). La desprofesionalización docente en educación especial. Revista Latinoamericana de Educación Inclusiva, 4(1), 105-126.

Hegarty, S. (2008). Investigación sobre educación especial en Europa. Revista Iberoamericana sobre Calidad, Eficacia y Cambio en Educación, 2(6), 119-199. Recuperado de https://revistas. uam.es/index.php/reice/article/view/5449 
Heitele, D. (1975). An epistemological view on fundamental stochastic ideas. Educational Studies in Mathematics, 6(2), 187-205. https://doi. org/10.1007/bf00302543

Ley General para las Personas con Discapacidad (2008). Diario Oficial de la Federación. México.

Maturana, H. (2003). Desde la biología a la psicología. Barcelona: Lumen.

Ojeda, A. M. (1994). Understanding fundamental ideas of probability at pre-university levels. Tesis de Doctorado inédita. King's College London.

Ojeda, A. M. (2006). Estrategia para un perfil nuevo de docencia: un ensayo en la enseñanza de estocásticos. En E. Filloy (ed.), Matemática educativa, treinta años (pp. 195-214). México D. F.: Santillana-Cinvestav.

Piaget, J. (1982). Le possible et le nécéssaire. París: Presses Universitaires de France (PUF).

Romero, S. y García, I. (2013). Educación especial en México. Desafíos de la educación inclusiva. Revista Latinoamericana de Educación Inclusiva, 7(2), 77-91.

Secretaría de Educación Pública (2004). Licenciatura en Educación Especial. Programa para la
Transformación y el Fortalecimiento Académicos de las Escuelas Normales. Plan de estudios. México D. F.: SEP.

Secretaría de Educación Pública (2006). Libro de matemáticas segundo grado. México D. F.: SEP.

Steinbring, H. (2005). The Construction of new Mathematical Knowledge in Classroom Interaction. Nueva York: Springer.

Tversky, A. y Kahneman, D. (1982). Availability: A heuristic for judging frequency and probability. En D. Kahneman, P. Slovic y A. Tversky (eds.), Judgment under Uncertainty: Heuristics and Biases. Cambridge, Reino Unido: Cambridge University Press. https://doi.org/10.1017/ cbo9780511809477.012

Unesco (1994). World Conference on Special Needs Education: Access and Quality, Salamanca, Spain. The Salamanca Statement and Framework for Action on Special needs Education. París.

Vygotski, L. S. (1997). Fundamentos de la defectología. Obra Escogidas V. Madrid: Aprendizaje Visor. 